\title{
Alterations in Xenobiotic-Metabolizing Enzyme Activities across Menstrual Cycle in Healthy Volunteers ${ }^{\mathrm{S}}$
}

\author{
E. Asprodini, ${ }^{1}$ V. Tsiokou, ${ }^{1}$ E. Begas, T. Kilindris, E. Kouvaras, M. Samara, and I. Messinis \\ Laboratory of Pharmacology (E.A., V.T., E.B., E.K.), Medical Informatics (T.K.), Pathology (M.S.), and Department of Obstetrics \\ and Gynecology (I.M.), Faculty of Medicine, School of Health Sciences, University of Thessaly, Larissa, Greece
}

Received October 10, 2018; accepted December 6, 2018

\begin{abstract}
The purpose of the study was to determine whether the in vivo activities of drug-metabolizing enzymes CYP1A2 and CYP2A6, xanthine oxidase (XO), and $\mathrm{N}$-acetyltransferase-2 (NAT2) vary across the menstrual cycle. Forty-two healthy women were studied at early follicular phase (EFP: 2nd to 4th days), late follicular phase (LFP: 10th to 12th days), and luteal phase (LP: 19th to 25th days) of a single menstrual cycle, and blood and urine samples were collected at each phase. Spot urine samples obtained 6 hours following 200-mg caffeine administration were used to determine caffeine metabolite ratios (CMRs); blood samples were used to determine CYP1A2*1F (rs762551) and CYP1A2*1C (rs2069514) polymorphisms and the hormonal profile (estradiol, progesterone, and luteinizing and follicle-stimulating hormones) at EFP, LFP, and LP. CMR and hormone variations were analyzed at three levels (EFP, LFP, LP) using one-way repeated-measures analysis of
\end{abstract}

variance. CYP1A2 activity was lower and that of CYP2A6 and NAT2 were higher at LFP compared with EFP and LP. Enzyme alterations were significant in volunteers $(n=21)$ whose hormonal profiles at EFP, LFP, and LP corresponded to expected levels, but not in volunteers $(n=15)$ with presumed early or late sampling around LFP. No significant difference was detected in any enzyme activity in presumed anovulatory volunteers $(n=6)$. The reduction of CYP1A2 activity at LFP was not associated with smoking or CYP1A2*1F polymorphism. XO and NAT2 (fast acetylators) activities remained unaltered. It is suggested that drug-metabolizing enzyme activities are altered across the menstrual cycle. Selection of appropriate sampling periods verified by hormonal assessment and identification of anovulatory cycles are decisive factors in disclosing altered enzyme activity across the menstrual cycle.

\section{Introduction}

Hormonal fluctuations during a menstrual cycle have been postulated to align with disease exacerbation as in catamenial epilepsy (Herzog, 2008) and perimenstrual asthma (Graziottin and Serafini, 2016). These fluctuations may also influence the treatment outcome by modifying drug pharmacokinetics and/or pharmacodynamics. Enhanced metabolism has been reported for phenytoin during menstruation (Shavit et al., 1984), methaqualone during ovulation (Wilson et al., 1982), omeprazole during both menstruation and the luteal phase (Nazir et al., 2015), and methylprednisolone during the luteal phase (Lew et al., 1993); reduced metabolism has been reported for theophylline (Bruguerolle et al., 1990) and paracetamol during the luteal phase of the menstrual cycle (Wójcicki et al., 1979).

The influence exerted by reproductive hormones on xenobioticmetabolizing enzymes is supported by studies showing that CYP1A2 activity is inhibited by estrogen-containing oral

The study was financially supported by the Research Committee of the University of Thessaly [Grant 4822].

${ }^{1}$ E.A. and V.T. contributed equally to this work.

https://doi.org/10.1124/jpet.118.254284.

S This article has supplemental material available at jpet.aspetjournals.org. contraceptives (OCs) (Granfors et al., 2005), estrogen replacement therapy (Pollock et al., 1999; O'Connell et al., 2006), and pregnancy (Vistisen et al., 1992; Tsutsumi et al., 2001; Tracy et al., 2005); CYP2A6 activity is enhanced in pregnancy (Dempsey et al., 2002), or during the use of estrogen-only OCs (Benowitz et al., 2006), whereas the effect of pregnancy (Tsutsumi et al., 2001) or estrogen therapy (Shelepova et al., 2005; O'Connell et al., 2006) on $N$-acetyltransferase-2 (NAT2) and xanthine oxidase (XO) activity has been poorly explored. With respect to the activity of drug-metabolizing enzymes across the menstrual cycle, available evidence is sparse and conflicting, as both reduced (Bruguerolle et al., 1990; Lane et al., 1992; Nagata et al., 1997) and no effect (Kashuba et al., 1998; Zaigler et al., 2000; Hukkanen et al., 2005) have been reported. These conflicting results are most likely due to suboptimal study designs based on small numbers of women, lack of characterization of menstrual cycle phases through assessment of hormonal concentrations in plasma, and, most importantly, lack of sampling at the time of the highest hormonal fluctuation such as that occurring at late follicular phase (LFP) (Fig. 1). In fact, due to the retrospective nature of menstrual cycle protocols and the variability of a normal menstrual cycle, menstrual cycle studies have an inherent

ABBREVIATIONS: AFMU, 5-acetylamino-6-formylamino-3-methyluracil; ANOVA, analysis of variance; 95\% Cl, 95\% confidence interval; CMR, caffeine metabolic ratio; EFP, early follicular phase; FSH, follicle-stimulating hormone; LFP, late follicular phase; LH, luteinizing hormone; LP, luteal phase; NAT2, $\mathrm{N}$-acetyltransferase-2; OC, oral contraceptive; PCR, polymerase chain reaction; 1U, 1-methyluric acid; 17U, 1,7-dimethyluric acid; 137X, 1,3,7-trimethylxanthine; 1X, 1-methylxanthine; 17X, 1,7-dimethylxanthine; XO, xanthine oxidase. 
A

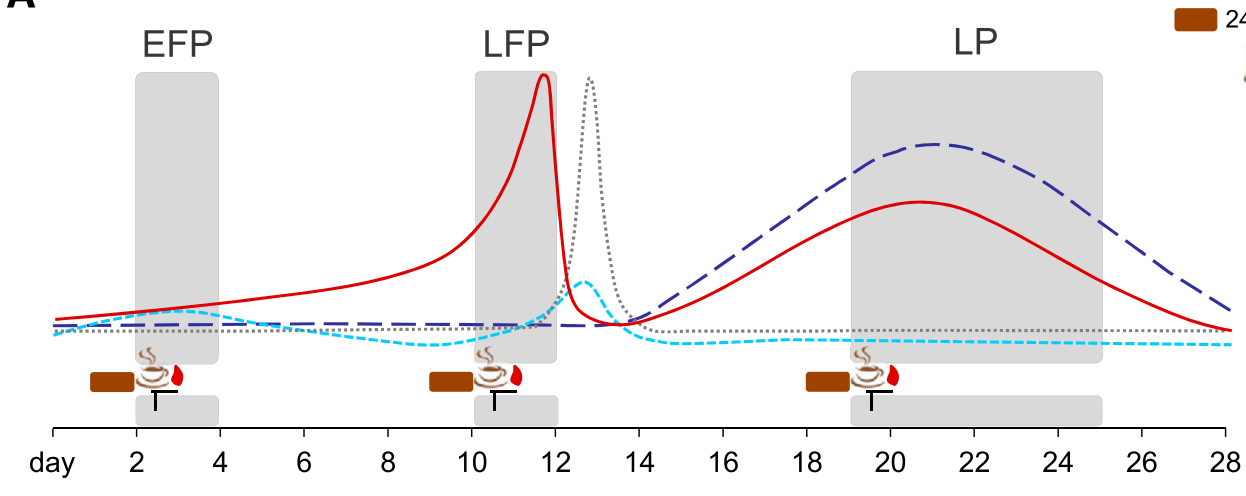

[IIIII sampling phase $24 \mathrm{~h}$ caffeine abstinence 3) caffeine test \& T blood sampling

B Reduced CYP1A2 activity

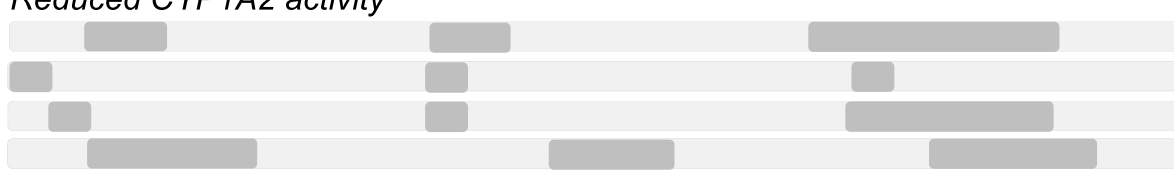

No effect

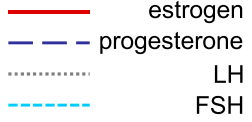

$\mathrm{FSH}$

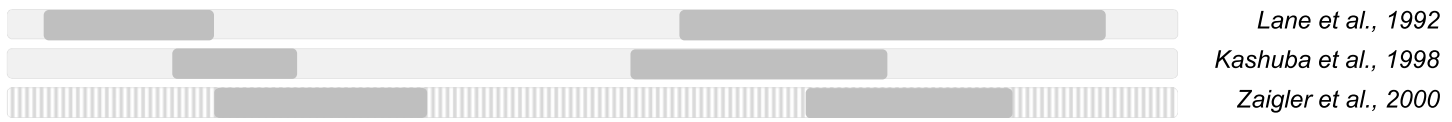

Fig. 1. Sampling phases in experimental protocols studying enzyme activity during the menstrual cycle. (A) Schematic diagram of the experimental protocol followed in the present study across one menstrual cycle. Samples were collected at three sampling phases designated by the gray shaded areas: EFP, 2nd to 4th days; LFP, 10th to 12th days; and LP, 19th to 25th days post onset of menses. Blood and urine samples were collected at each phase 6 hours after the caffeine test, following 24-hour abstinence from caffeine-containing foods and beverages (box preceding coffee cup). Reference levels of estradiol, progesterone, LH, and FSH during menstrual cycle are shown in different line types. (B) Literature review of sample collection phases determining xanthine pharmacokinetics across menstrual cycle. Reduced CYP1A2 activity was reported in studies which included LFP sampling in their experimental protocol and compared EFP and LP to LFP. Conversely, no effect in enzyme activity was reported in studies which were designed to make comparisons between follicular and luteal phases.

difficulty identifying the precise timing of peak and trough hormone levels that may influence the activity of drugmetabolizing enzymes, unlike studies on OCs, hormonal replacement therapy or pregnancy in which sampling occurs at presumed steady-state hormone levels.

The activity of drug-metabolizing enzymes, including CYP1A2, CYP2A6, XO, and NAT2, has long been assessed through molar ratios of the different caffeine metabolites (Fig. 2) (Asprodini et al., 1998; Begas et al., 2007; Hakooz, 2009). Human CYP1A2 has the highest catalytic activity in the 2-hydroxylation of estradiol (Yamazaki et al., 1998) and is responsible for the metabolism of many clinically used drugs (Faber et al., 2005). CYP2A6 catalyzes the biotransformation of nicotine and drugs such as valproic acid; $\mathrm{XO}$ oxidizes endogenous purines and pyrimidines and metabolizes drugs such as thiopurines and methylxanthines. Similarly, NAT2 is involved in the acetylation of many drugs (Evans, 1989).

The aim of the present study was to examine the activity of CYP1A2, CYP2A6, XO, and NAT2 enzymes in three sampling phases of the menstrual cycle [EFP, LFP, and luteal phase (LP)] in healthy women, using caffeine as a metabolic probe. The menstrual status of each volunteer was verified upon evaluation of the hormonal profile of estradiol, progesterone, luteinizing hormone ( $\mathrm{LH}$ ), and follicle-stimulating hormone (FSH) concentrations in blood. Moreover, the effect of genotype on the phenotypic expression of CYP1A2 across the menstrual cycle was investigated by examining CYP1A2 caffeine metabolic ratios $(\mathrm{CMRs})$ in relation to the two most common CYP1A2 single nucleotide polymorphisms, CYP1A2*1C and CYP1A21*F.

\section{Materials and Methods}

Subjects. The population study consisted of 42 (25 nonsmokers, 17 smokers) apparently healthy female volunteers with regular menstrual cycles. Their health status was based on medical history and physical examination and was confirmed by routine laboratory tests (aspartate aminotransferase, alanine aminotransferase, alkaline phosphatase, $\gamma$-glutamyl transpeptidase, serum creatinine, and serum urea; Table 1). Exclusion criteria were the use of OCs (or any hormonal birth control method), pregnancy, menstrual cycle irregularities, medications known to induce or inhibit the activity of the enzymes of interest, and consumption of alcohol. A form regarding demographic and lifestyle data (age, weight, height, regularity and duration of menstrual cycle, chronic diseases, medication intake, smoking and alcohol consumption habits, occupation, and exposure to xenobiotics) was completed for all participants. A detailed description of inclusion/exclusion criteria, the study protocol, and data collection is provided in the Supplemental Methods.

The study was carried out in accordance with the Declaration of Helsinki and approved by the Ethics Committee of Larissa University Hospital, Greece (1862015/24227). Written informed consent was obtained from all subjects before they entered the study.

Study Protocol. Blood and spot urine samples were collected during a single menstrual cycle at days corresponding to three sampling phases: EFP (2nd to 4th days), LFP (10th to 12th days), and LP (19th to 25 th days) (Fig. 1A); expected hormone levels at EFP, $\mathrm{LFP}$, and LP were defined according to reference concentration ranges reported in the literature (Reed and Carr, 2000).

Volunteers were asked to abstain from methylxanthine-containing food or beverages for at least 24 hours and to refrain from cruciferous and apiaceous vegetables (Kall et al., 1996; Lampe et al., 2000; Peterson et al., 2009), grapefruit juice (Fuhr et al., 1993), and broiled meat (Kall et al., 1996; Perera et al., 2012) for 48 hours before the 


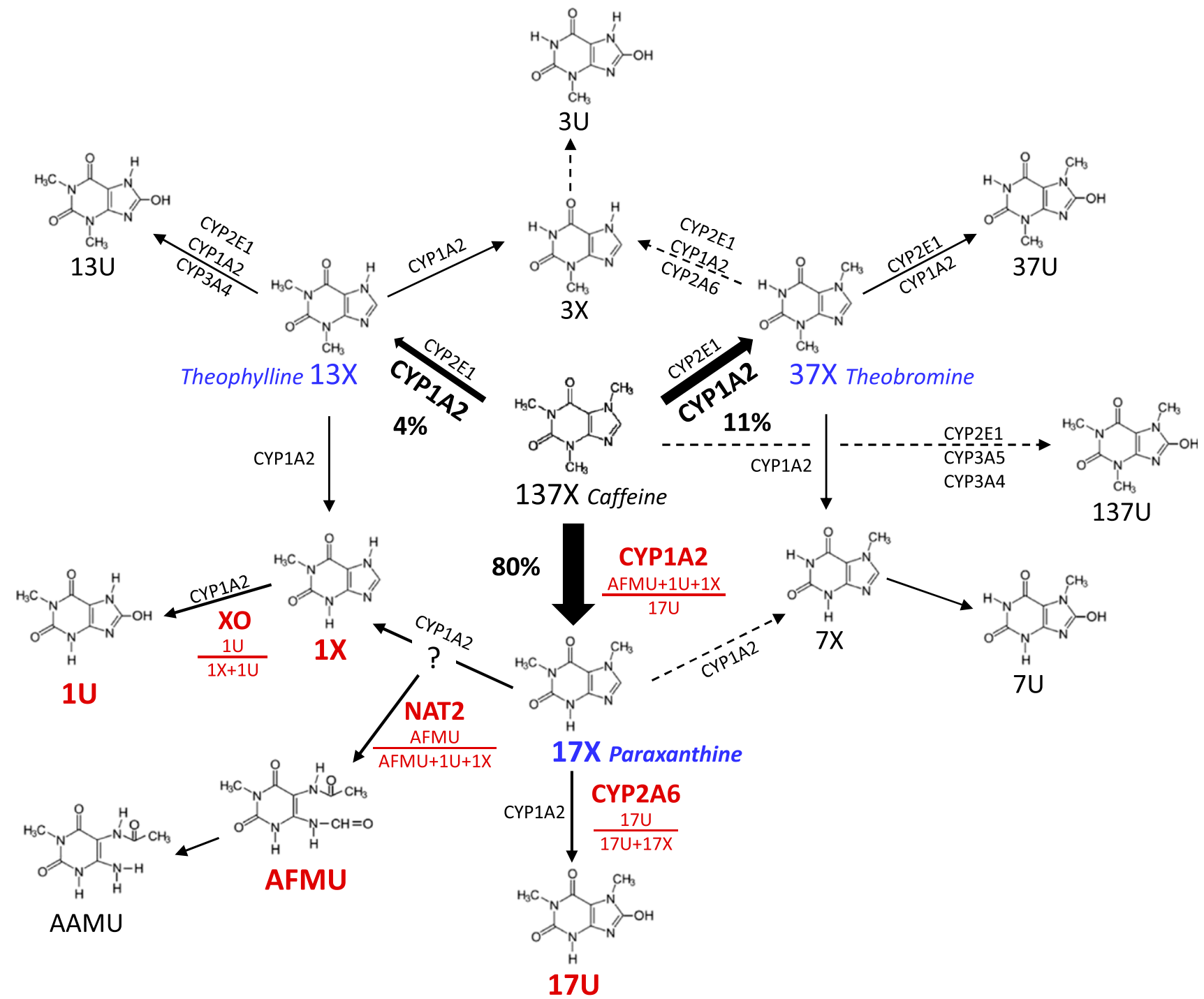

Fig. 2. Metabolic pathways involved in caffeine metabolism in humans. The major pathway in the metabolism of caffeine is catalyzed by CYP1A2 and involves the $N-1, N-3$, and $N-7$ demethylations of caffeine [1,3,7-trimethylxanthine (137X)] to form theobromine [3,7-dimethylxanthine (37X)], paraxanthine [1,7-dimethylxanthine (17X)], and theophylline [1,3-dimethylxanthine (13X)], respectively (shown in blue), accounting for about 80\%, 11\%, and $4 \%$ of caffeine metabolism (heavy arrows) (Lelo et al., 1986; Gu et al., 1992). Dimethylxanthines are $N$-demethylated to the corresponding monomethylxanthine, 1-methylxanthine (1X), 3-methylxanthine (3X), and 7-methylxanthine (7X). Caffeine and xanthines are hydroxylated into their corresponding uric acids: 1,3,7-trimethyluric acid (137U), 1,3-dimethyluric acid (13U), 1,7-dimethyluric acid (17U), 3,7-dimethyluric acid (37U), 1-methyluric acid (1U), 3-methyluric acid (3U), and 7-methyluric acid (7U). CYP2A6 catalyzes the conversion of paraxanthine to 17U. The polymorphic enzyme NAT2 catalyzes the C8-N9 bond scission and the acetylation of paraxanthine to produce AFMU, which is then converted nonenzymatically into 5-acetylamino-6-amino-3-methyluracil (AAMU) in urine. XO is responsible for the conversion of 1X into 1U. Metabolites, enzymes, and metabolic molar ratios used as indices of enzyme activities in the present study are shown in red. Dashed arrows indicate minor metabolic pathways.

caffeine test. On the day of the test, volunteers were administered a 200-mg caffeine capsule. Spot urine samples were collected 6 hours later in containers preloaded with $200 \mu \mathrm{l}$ of $6 \mathrm{~N} \mathrm{HCl}$ (Fig. 1A).

Chemicals. Caffeine metabolites 1,7-dimethylxanthine (17X), 1,7dimethyluric acid (17U), and 1-methyluric acid (1U) were purchased from Sigma-Aldrich (Steinheim, Germany); 1-methylxanthine (1X) was purchased from TCI (Zwijndrecht, Belgium); and 1,3,7-trimethylxanthine (137X) was from Fluka (Buchs, Switzerland). 5-Acetylamino-6-formylamino3-methyluracil (AFMU) was kindly provided by Wolfgang Pfleiderer (University of Konstanz, Konstanz, Germany). Chloroform and isopropanol were purchased from Chem Lab (Zedelgem, Belgium) and Honeywell Research Chemicals (Seelze, Germany), respectively.

Sample Analysis. Urinary caffeine metabolites were quantified by reversed-phase high-performance liquid chromatography as previously described (Begas et al., 2007). Urine samples were acidified to $\mathrm{pH}=3.5$ to ensure AFMU stability (Wong et al., 2002); 1-ml aliquots were stored at $-20^{\circ} \mathrm{C}$ until analysis. Caffeine metabolites were isolated from urine samples by liquid-liquid extraction using chloroform/ isopropanol. Calibration curves for caffeine metabolites in urine were linear at concentrations of $10-500 \mu \mathrm{M}$ with $R^{2}>0.99$. Interday precision was $90.38 \%-97.70 \%$ and accuracy was $94.04 \%-105.65 \%$ at concentrations of 25,150 , and $400 \mu \mathrm{M}(n=10)$. The low limit of quantitation for metabolites was $5 \mu \mathrm{M}$, and the limits of detection were $0.08,0.03,0.09,0.10$, and $0.17 \mu \mathrm{M}$ for AFMU, $1 \mathrm{U}, 1 \mathrm{X}, 17 \mathrm{U}$, and $17 \mathrm{X}$, respectively. In vivo evaluation of enzyme activities was assessed using the CMRs as follows: CYP1A2 $=(\mathrm{AFMU}+1 \mathrm{U}+1 \mathrm{X}) / 17 \mathrm{U}$, $\mathrm{CYP} 2 \mathrm{~A} 6=17 \mathrm{U} /(17 \mathrm{U}+17 \mathrm{X}), \mathrm{XO}=1 \mathrm{U} /(1 \mathrm{X}+1 \mathrm{U})$, and NAT2 $=\mathrm{AFMU} /$ $(\mathrm{AFMU}+1 \mathrm{U}+1 \mathrm{X})$ (Begas et al., 2007).

Estradiol, progesterone, LH, and FSH concentrations were determined by electrochemiluminescence immunoassay (Cobas e 411 analyzer, Roche Diagnostics GmbH D-68298 Mannheim Germany). According to the manufacturer, the precisions of the assays for 
TABLE 1

Demographic characteristics and biochemical data of the participants ( $n=42$; nonsmokers, $n=26$; smokers, $n=16$ )

\begin{tabular}{|c|c|c|c|c|c|c|c|}
\hline \multirow{2}{*}{ Variable } & \multicolumn{2}{|c|}{ All } & \multicolumn{2}{|c|}{ Nonsmokers } & \multicolumn{2}{|c|}{ Smokers } & \multirow{2}{*}{ Reference value } \\
\hline & Median & Range & Median & Range & Median & Range & \\
\hline Age (years) & 30.5 & $18-45$ & 30 & $18-45$ & 32 & $18-44$ & \\
\hline Weight $(\mathrm{kg})$ & 63 & $46-95$ & 62 & $46-95$ & 63 & $53-90$ & \\
\hline Body mass index $\left(\mathrm{kg} / \mathrm{m}^{2}\right)$ & 22.4 & $17.5-40.6$ & 21.9 & $17.5-40.6$ & 22.8 & $18.6-33.9$ & \\
\hline Duration of menstrual cycle (days) & 30 & $27-33$ & 28 & $27-30$ & 28 & $28-35$ & \\
\hline \multicolumn{8}{|l|}{ Sampling days } \\
\hline EFP & 3 & $2-4$ & 3 & $2-4$ & 3 & $2-4$ & \\
\hline LFP & 11 & $10-12$ & 11 & $10-12$ & 11 & $10-12$ & \\
\hline LP & 22 & $19-25$ & 22 & $19-25$ & 22 & $20-24$ & \\
\hline Aspartate aminotransferase (IU/l) & 15.0 & $9.0-32.0$ & 15.0 & $10.0-24.0$ & 18.0 & $9.0-32.0$ & $5.0-32.0$ \\
\hline Alanine aminotransferase (IU/l) & 11.0 & $7.0-40.0$ & 11.0 & $7.0-18.0$ & 11.5 & $7.0-28.0$ & $5.0-41.0$ \\
\hline Alkaline phosphatase (IU/l) & 57.5 & $39.0-84.0$ & 55.0 & $35.0-84.0$ & 63.5 & $40.0-78.0$ & $40.0-129.0$ \\
\hline$\gamma$-Glutamyl transpeptidase (IU/l) & 11.0 & $7.0-23.0$ & 9.0 & $7.0-19.0$ & 12.5 & $8.0-23.0$ & $5.0-39.0$ \\
\hline Serum creatinine $(\mathrm{mg} / \mathrm{dl})$ & 0.70 & $0.49-0.97$ & 0.70 & $0.56-0.97$ & 0.68 & $0.49-0.87$ & $0.50-0.90$ \\
\hline Serum urea $(\mathrm{mg} / \mathrm{dl})$ & 22.0 & $14.0-43.0$ & 26.0 & $14.0-43.0$ & 21.5 & $14.0-27.0$ & $15.0-50.0$ \\
\hline
\end{tabular}

estradiol, progesterone, $\mathrm{LH}$, and FSH were $2.5 \%-11.9 \%, 3.7 \%-5.5 \%$, $1.9 \%-5.2 \%$, and $2.9 \%-5.3 \%$, respectively. Venous blood samples were collected, centrifuged and stored at $-20^{\circ} \mathrm{C}$ until analysis.

CYP1A2 Genotype Analysis. Peripheral blood was collected from each subject, and genomic DNA was extracted using a Purelink genomic DNA mini kit (Invitrogen, Carlsbad, CA). Genotyping was performed by the polymerase chain reaction (PCR) restriction fragment length polymorphism method. Four microliters of DNA was added in each tube containing $1 \times$ buffer, $1.6 \mathrm{mM}$ deoxynucleotide triphosphates (dNTPs), $2 \mathrm{mM} \mathrm{MgCl} 2,400 \mathrm{nM}$ each primer, and $1.5 \mathrm{U}$ of Taq DNA polymerase (Fermentas Inc., Hanover, MD) in a total volume of $50 \mu$ l. Digestion was performed by BsII and PspOMI restriction enzymes for rs2069514 $(-3860 \mathrm{G}>\mathrm{A})$ and rs762551 $(-163 \mathrm{C}>\mathrm{A})$ Single Nucleotide Polymorphisms (SNPs), respectively. (PCR conditions and specific primers' sequences are shown in Table 2.) PCR and digestion products were evaluated by agarose gel electrophoresis under ethidium bromide staining (Fig. 4b1).

Statistical Analysis. Fluctuations in hormone (estradiol, progesterone, LH, and FSH) concentrations and CMRs were compared at three levels (EFP, LFP, and LP) using one-way repeated-measures analysis of variance (ANOVA). CYP1A2 CMRs were compared between smokers and nonsmokers within the three sampling phases using mixed repeated-measures ANOVA. Specifically, CYP1A2 CMRs were compared across EFP, LFP, and LP within subjects, using the $\mathrm{A} / \mathrm{A}$ and $\mathrm{C} / \mathrm{A}$ genotypes and the nonsmoking/smoking habits of the subjects as control factors; repeated contrasts were used to test for significant differences among sampling phases. The levels of the factor genotype were reduced to two, as genotype $\mathrm{C} / \mathrm{C}$ was encountered in only one subject. Results are reported as estimated marginal means for each level with 95\% confidence intervals (95\% CIs) and as parameter estimates for subgroups (mean values \pm S.E.). CYP2A6, $\mathrm{XO}$, and NAT2 CMRs were compared within the three sampling phases using one-way repeated-measures ANOVA. Multiple pairwise comparisons in ANOVA were $P$-corrected using Bonferroni adjustment. The statistical difference between two independent groups was examined by $t$ test. All statistical analyses were performed using SPSS version 24 software (IBM, Armonk, NY). $P$ values $<0.05$ were considered statistically significant.

\section{Results}

Forty-two women met the inclusion criteria and were enrolled in the study; demographic characteristics and biochemical data of the participants are presented in Table 1. No subject reported adverse effects after the caffeine test.

Hormone Levels during Menstrual Cycle. Estradiol, progesterone, LH, and FSH blood concentrations were measured at the EFP, LFP, and LP (Fig. 1A, gray-shaded blocks; Supplemental Table 1). Upon completion of the study protocol, the concentration profile of all hormones was compared with the profile of reference concentrations reported in the literature (Reed and Carr, 2000) (depicted with solid red, dashed purple, dotted gray, and dashed cyan lines, respectively, within gray blocks in Fig. 1A). In 21 volunteers (21/42, $50 \%$ ), serum hormonal concentrations determined at EFP, LFP, and LP corresponded to expected hormone levels (group 1; red solid line within gray blocks in Fig. 1A), such that the concentrations of estradiol and LH peaked at LFP in relation to EFP and LP (Fig. 3; Table 3). In 15 volunteers (15/42, $35.7 \%$ ), estradiol and LH levels at LFP did not concur with the expected levels presumably due to earlier or later sampling relative to hormone concentration peaks at LFP (group 2). Six volunteers $(6 / 42,14.3 \%)$ were considered to have anovulatory menstrual cycles (group 3), as their serum progesterone level, measured at LP, was $<2.5 \mathrm{ng} / \mathrm{ml}$ (Prior et al., 2015) (Fig. 3; Table 3). Conversely, serum progesterone concentration was higher in LP compared with EFP and LFP in groups 1 and 2. FSH levels at LP were significantly lower compared with EFP and LFP in groups 1 and 2. Group 3 did not exhibit significant differences in any hormone concentration among EFP, LFP, and LP.

In Vivo Activity of CYP1A2 during Menstrual Cycle. The effect of genotype and smoking on the phenotypic expression of CYP1A2 during the menstrual cycle was examined by comparing CYP1A2 CMRs at EFP, LFP, and LP among different genotypes in nonsmoking and smoking volunteers.

The frequencies of CYP1A2*1F (rs762551) polymorphism $(-163 \mathrm{C}>\mathrm{A})$ within the $\mathrm{C} / \mathrm{A}, \mathrm{A} / \mathrm{A}$, and $\mathrm{C} / \mathrm{C}$ genotypes were $24 / 42$ $(57.1 \%), 17 / 42(40.5 \%)$, and $1 / 42(2.4 \%)$, respectively. These frequencies did not differ significantly from the values predicted by the Hardy-Weinberg equilibrium model ( $\chi^{2}$ test, $P=0.084$ ). The $\mathrm{G}>\mathrm{A}$ polymorphism in the -3860 position (CYP1A $2 * 1 \mathrm{C}$, rs2069514) was not detected, in accordance with other Caucasian populations (Dobrinas et al., 2011). CYP1A2 CMRs, examined at EFP $(n=42)$, were similar between C/A and A/A genotypes in nonsmokers; conversely, smokers homozygous for the A allele exhibited a trend for higher CMRs compared with heterozygous C/A, suggesting that the A/A genotype confers higher CYP1A2 inducibility (Fig. 4b2, b3), as has been previously reported [Sachse et al. (1999) and Gunes et al. (2009), but see Dobrinas et al. (2011)]. 
In group 1 (genotypes C/A and A/A, $n=20$ ), CYP1A2 CMR was significantly lower (23\%) at LFP compared with EFP and LP; no significant difference was detected between EFP and LP, suggesting that CMR recovers at LP to the baseline EFP level (Fig. 4A; Table 3). CYP1A2 CMRs were $13.6 \%$ and $24.3 \%$ lower at LFP compared with EFP in nonsmokers and smokers, respectively. CYP1A2 CMRs were increased (7.3\% and 9.9\%) and remained almost the same (1.1\% and $2.4 \%$ reduction) in four nonsmokers at LFP compared with EFP; the rest exhibited an 18.5\% decrease (range: 5.7\%-32.0\%). Correspondingly, the metabolic ratio was increased by $26.3 \%$ and remained almost the same ( $1.1 \%$ reduction) in two smokers at LFP compared with EFP; the rest of the smokers exhibited a $27.7 \%$ decrease (range: 5.6\%-52.9\%), indicating large interindividual variation.

Group 2 exhibited reduced CYP1A2 CMRs at LFP compared with LP (but not EFP), probably due to a slight shift in sampling, either earlier or later, relative to estradiol peak at LFP (see Discussion). No significant difference was detected in CYP1A2 CMR between EFP and LP, suggesting that CMR in LP recovers to the baseline EFP level.

Group 3 exhibited a nonsignificant difference in CYP1A2 CMRs among EFP [3.92 (95\% CI: 3.03-4.80)], LFP [4.12 (95\% CI: 3.00-5.24)], and LP [3.51 (95\% CI: 2.81-4.22)] (Table 3).

In all groups, neither genotype nor smoking exhibited a significant interaction with sampling phase.

CYP1A2 CMRs at EFP $(n=42)$ were significantly lower in nonsmokers compared with smokers, confirming the inducing effect of smoking on CYP1A2 activity.

In Vivo Activities of CYP2A6, XO, and NAT2 during Menstrual Cycle. Previous studies have shown that smoking does not affect CYP2A6 (Nowell et al., 2002; Begas et al., 2007), XO (Chung et al., 2000; Aklillu et al., 2003; Benowitz et al., 2003; Begas et al., 2007), and NAT2 activity (Benowitz et al., 2003; Begas et al., 2007). Therefore, smoking was not included as a factor in the analysis of CYP2A6, XO, and NAT2 CMRs among menstrual sampling phases.

In group 1, CYP2A6 CMRs were significantly higher at LFP compared with EFP and LP with no significant difference between EFP and LP, suggesting that CMR at LP recovers to the baseline EFP level. Similarly, group 2 exhibited higher CYP2A6 activity at LFP compared with EFP and LP, although statistical significance was reached only between LFP and LP. Group 3 exhibited CYP2A6 CMRs that did not differ significantly among EFP, LFP, and LP. XO CMRs did not differ among the three sampling phases of the menstrual cycle in all groups studied (Fig. 5; Table 3).

Volunteers were classified as slow $(n=27 / 42,64.3 \%)$ and fast ( $n=15 / 42,35.7 \%$ ) acetylators according to the antimode of 0.25 (Begas et al., 2007). Slow acetylators in group 1 exhibited significantly higher NAT2 CMRs at LFP compared with EFP and LP. No significant difference was detected in NAT2 CMRs of fast acetylators in group 1 and slow and fast acetylators in groups 2 and 3 among menstrual sampling phases (Fig. 5; Table 3).

\section{Discussion}

The present study is the first to show that CYP1A2 CMR is significantly reduced at LFP compared with EFP and recovers at LP; the reduction at LFP is most prominent when sampling coincides with the estradiol peak and less prominent when it 


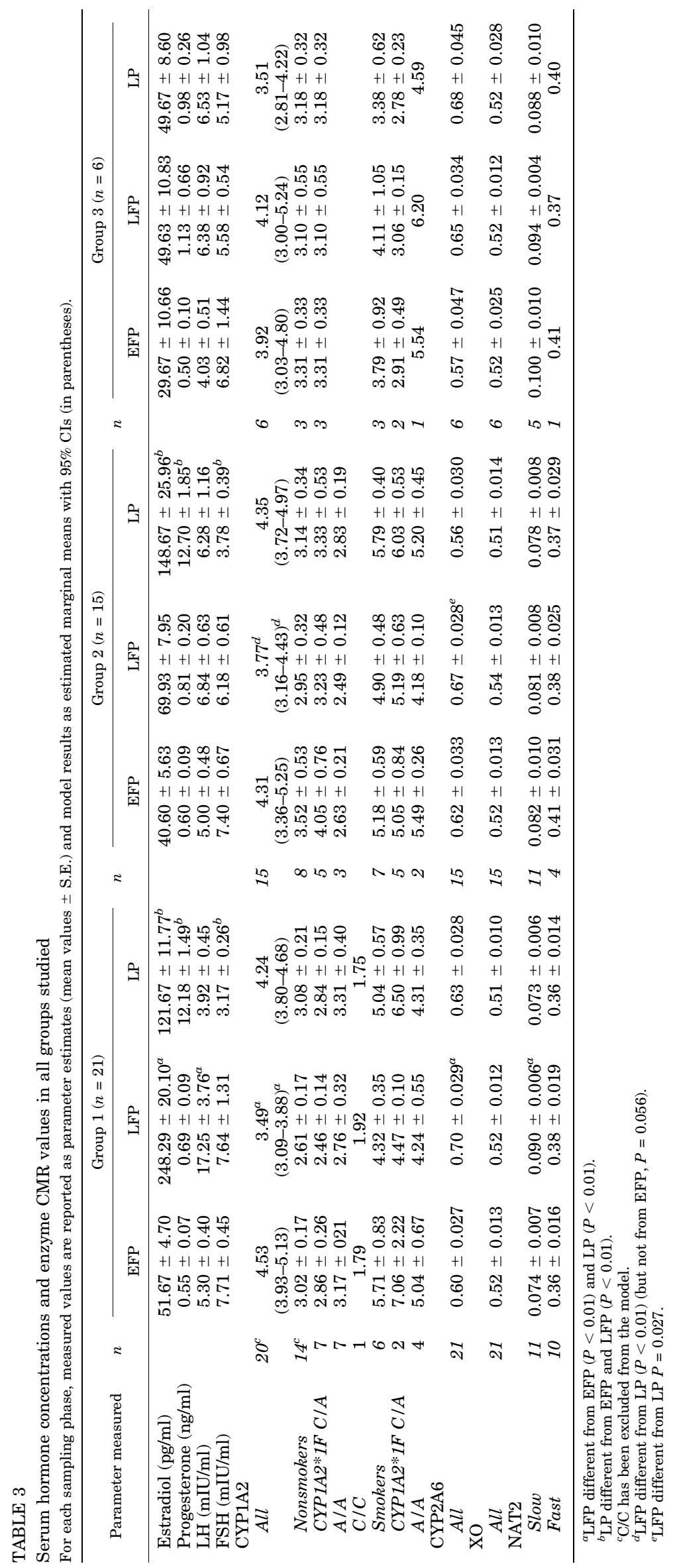



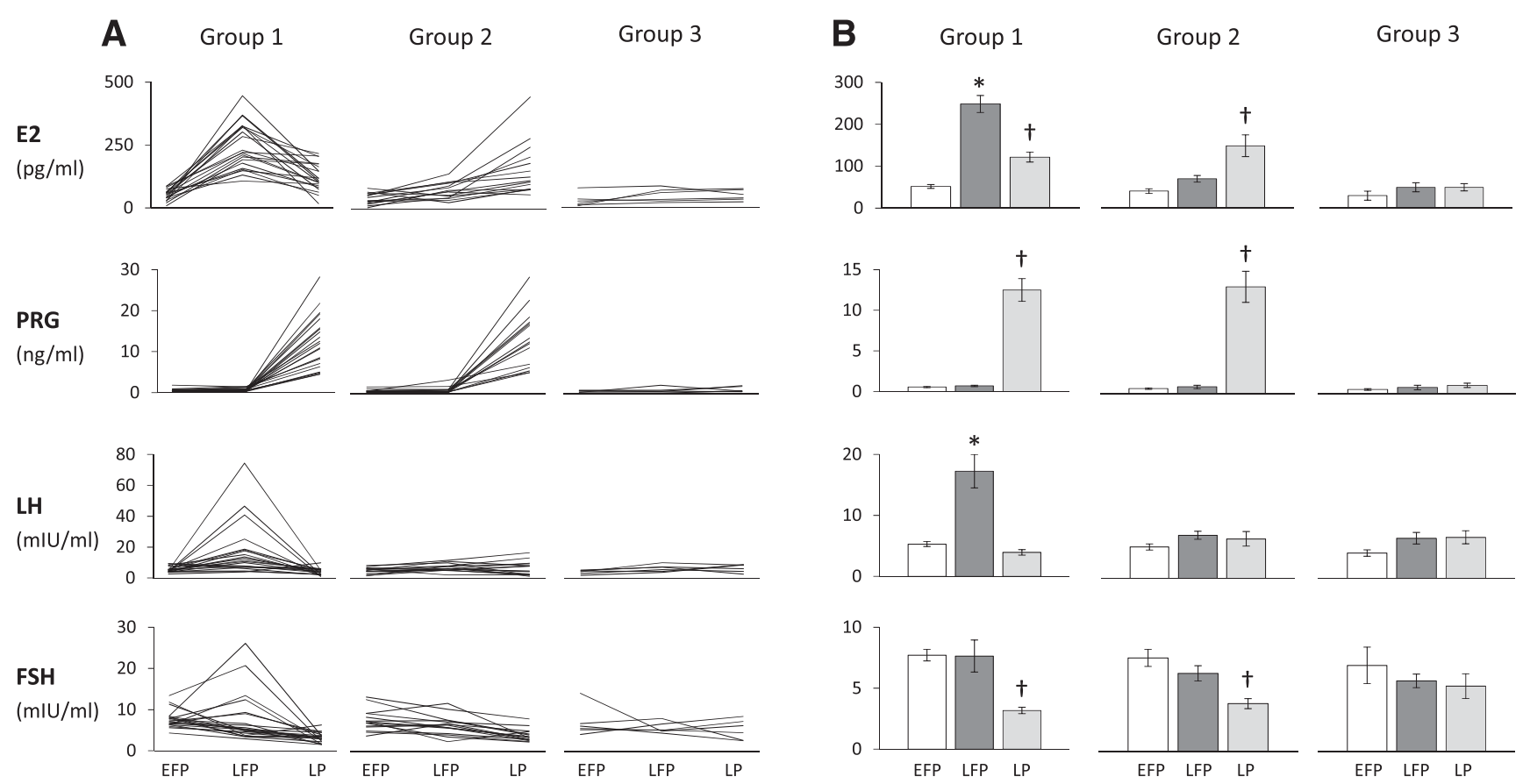

Fig. 3. Hormone levels during menstrual cycle. Individual data (A) and mean estimates ( \pm S.E.) (B) of estradiol (E2), progesterone (PRG), LH, and FSH in 42 healthy volunteers (group 1: $n=21$; Group 2: $n=15$; Group 3: $n=6$ ) at the three sampling phases (EFP, LFP, and LP). *EFP is different from LFP and LP; $\dagger$ LP is different from EFP and LFP. CMRs were compared across sampling phases at three levels (EFP, LFP, LP) using one-way repeatedmeasures ANOVA.

occurs earlier or later relative to the LFP estradiol peak. The reduction of CYP1A2 activity at LFP is not associated with smoking or CYP1A2*1F (rs762551) polymorphism. CYP1A2 CMR is not altered in presumed anovulatory cycles.

The effect of the menstrual cycle on CYP1A2 activity has been disputed over the past decades by several investigators (Fig. 1). Studies reporting no effect of the menstrual cycle on CYP1A2 activity based their conclusion upon comparisons between two sampling phases, namely EFP and LP; indeed, no effect of menstrual cycle on CYP1A2 activity, caffeine CMR (Kashuba et al., 1998), or caffeine clearance (Lane et al., 1992) was found between EFP and LP. Studies reporting altered CYP1A2 activity across the menstrual cycle based their conclusion upon comparisons among three sampling phases: EFP, LFP, and LP. Nagata et al. (1997) and Kamimori et al. (1999) reported that theophylline and caffeine metabolism, respectively, is reduced in the ovulatory (LFP) compared with the menstrual phase (EFP) and LP; they also reported that there is no difference in CYP1A2 activity between EFP and LP. Therefore, the dispute among studies on the effect of menstrual cycle on CYP1A2 activity is only apparent since all studies, including the present one, agree that CYP1A2 activity is unaltered when comparisons are made between EFP and LP. Consequently, it is possible that alterations of CYP1A2 activity across the menstrual cycle are unraveled only in those experimental protocols that use sampling at the late follicular phase, when steep alterations in estradiol levels occur-namely, either at the peak (present study, group 1; Kamimori et al., 1999) or around the peak of estradiol concentration (present study, group 2; Nagata et al., 1997; Kamimori et al., 1999). This hypothesis is supported by a recent report showing that, in ovulatory cycles, the most prominent increase in serum caffeine concentration is observed between early (day 7) and midfollicular (day 12) phases with a gradual recovery to baseline concentration during the luteal phase (Schliep et al., 2016). It is noteworthy that hormonal assessment throughout sampling facilitates the identification of anovulatory cycles, which are characterized by unaltered CYP1A2 activity (present study; Zaigler et al., 2000), and may contribute to underestimation of results when included in the sample (Kamimori et al., 1999). Overall, the present study provides evidence that selection of appropriate sampling phases, verification of menstrual phases by hormonal assessment, and identification of anovulatory cycles in study protocols may be decisive factors in disclosing an influence of the menstrual cycle on CYP1A2 activity.

Reduced CYP1A2 activity, with varied magnitude of reduction, has been reported following oral contraceptive use [20\%-55\% (Vistisen et al., 1992; Granfors et al., 2005)], estrogen replacement therapy in postmenopausal women [30\% (Pollock et al., 1999; O'Connell et al., 2006)], and in pregnancy [30\%-65\% (Vistisen et al., 1992; Tsutsumi et al., 2001; Tracy et al., 2005)]. Notably, women experience diverse patterns of exposure to estradiol in different conditions: sustained, yet low, estradiol concentration following exogenous administration of OCs [up to $50 \mathrm{pg} / \mathrm{ml}$ (Reape et al., 2008)] and hormonal replacement therapy [up to $150 \mathrm{pg} / \mathrm{ml}$ (Pollock et al., 1999; O'Connell et al., 2006)]; long-lasting incremental estradiol concentration reaching drastic levels during pregnancy [up to $6137 \mathrm{pg} / \mathrm{ml}$ (Abbassi-Ghanavati et al., 2009)]; and steep alteration in estradiol concentration occurring acutely during the menstrual cycle [up to $250 \mathrm{pg} / \mathrm{ml}$ at LFP (present study; Hambridge et al., 2013)]. Whether these different conditions share a common mechanism in reducing CYP1A2 activity, or whether estradiol is the only key player responsible for this reduction remains largely unknown. Nevertheless, as these studies have used caffeine as a metabolic probe, it is conceivable that the reduction in CYP1A2 activity could be attributed to pharmacologic interaction between estradiol and caffeine, as they are both substrates for cytochrome P450, although 
A Group 1

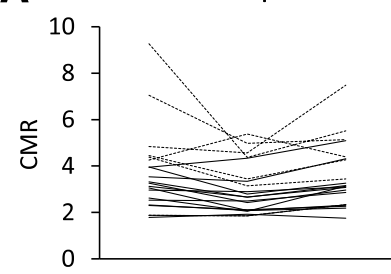

Group 2

Group 3
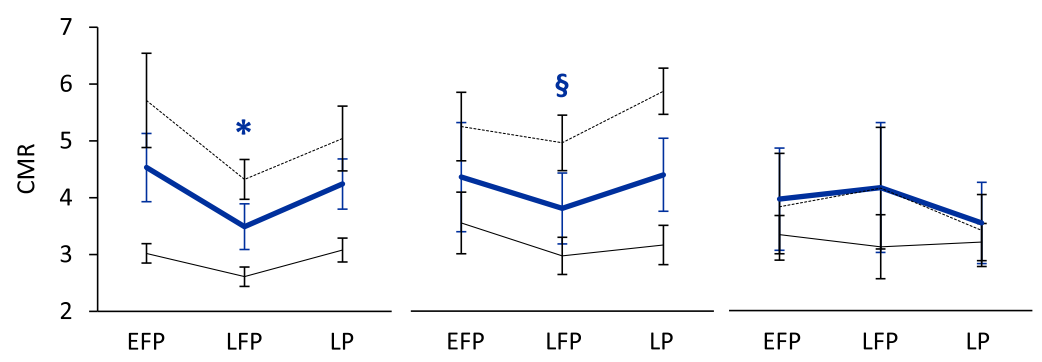

\section{B} $b_{1}$
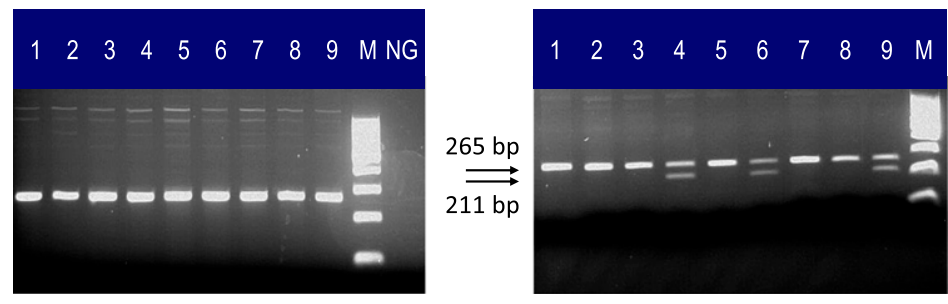

$b_{2}$
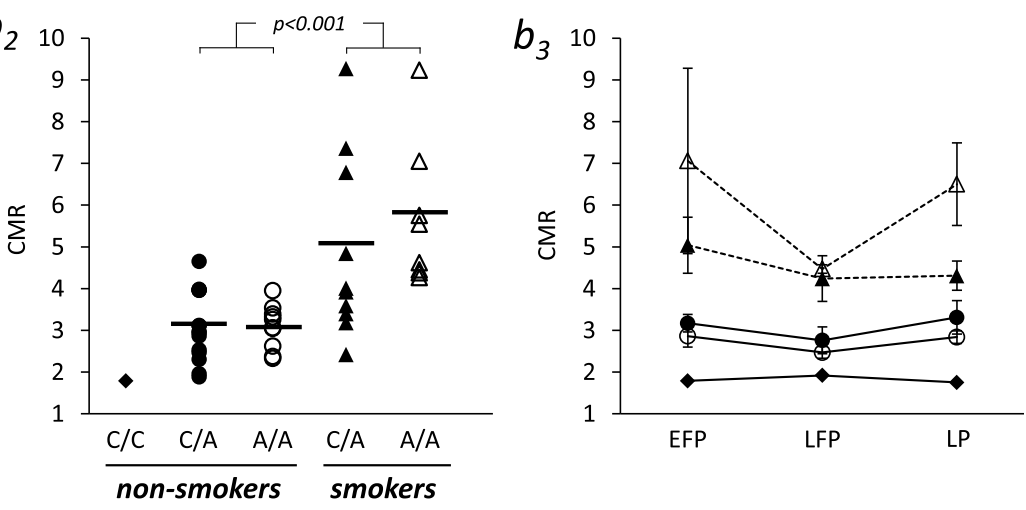

Fig. 4. CYP1A2 in vivo indices during menstrual cycle. (A) Individual data (upper graphs) and mean estimates ( \pm S.E., lower graphs) of CYP1A2 CMRs in nonsmoking (straight lines) and smoking (dashed lines) volunteers measured in the three groups of volunteers (group 1: $n=$ 21; group 2: $n=15$; group 3: $n=6$ ) at EFP, LFP, and LP. Thick solid lines in the lower graphs represent overall (nonsmoking and smoking) mean CYP1A2 CMR estimates (error bars denote 95\% CIs). *The overall CYP1A2 CMR value in group 1 is significantly reduced at LFP compared with $\operatorname{EFP}(P=0.002)$ and $\operatorname{LP}(P<0.001)$ (one-way repeated-measures ANOVA); §Overall CYP1A2 CMR value in group 1 is reduced at LFP compared with EFP $(P=0.056)$ and $\operatorname{LP}(P=0.003)$ (one-way repeated-measures ANOVA). Group 3 did not exhibit any significant difference in CYP1A2 CMRs among the three sampling phases. (B) PCR amplification products of genomic DNAs were extracted from peripheral blood (b1, left). M, 100-bp DNA ladder; NG, negative sample. PCR products were digested by PspOMI, and PCR restriction fragment length polymorphism of CYP1A2*1F (rs762551) were subjected to agarose gel electrophoresis (b1, right). A/A genotype: positions 1, 2, 3, 5, 7, 8; C/A genotype: positions 4, 6, 9. (b2) Scatter plot of CYP1A2 CMRs, considered at EFP, of all volunteers ( $n=42$ ) stratified by genotype and smoking. Mean CYP1A2 CMR values (horizontal lines) are higher in smokers compared with nonsmokers $(P<0.001 ; t$ test). Symbols for genotype are as follows: nonsmokers: diamonds for C/C $(n=1)$, filled circles for C/A $(n=15)$, open circles for A/A $(n=10)$; smokers: filled triangles for C/A $(n=9)$, open triangles for A/A $(n=7)$. (b3) Mean CYP1A2 CMR estimates ( \pm S.E.) at EFP, LFP, and LP in group $1(n=21)$. Neither genotype nor smoking exhibited any significant interaction with sampling phase; straight lines, nonsmokers $(n=15)$; dashed lines, smokers $(n=6)$. experimental evidence from in vitro studies has shown that estradiol is only a weak competitive inhibitor of CYP1A2 (Karjalainen et al., 2008; Chang et al., 2009). An alternative candidate mechanism could be transcriptional downregulation of the CYP1A gene, as has been shown previously for CYP1A1 and CYP2E1 (Beischlag and Perdew, 2005; Konstandi et al., 2013).

The higher CYP2A6 activity observed at LFP compared with EFP and LP is not surprising, as previous reports have associated enhanced CYP2A6 activity with conditions of increased estrogen levels, such as pregnancy (Dempsey et al., 2002), or the use of estrogen-only OCs (Benowitz et al., 2006). Furthermore, estradiol has been shown to increase the expression of CYP2A6 in human hepatocytes in vitro (Choi et al., 2013). Similar pharmacokinetics of nicotine, a substrate of CYP2A6, between the midfollicular and the midluteal phase have been reported, although the authors acknowledged the possibility of altered CYP2A6 activity during other phases, such as the ovulatory or menstrual phases (Hukkanen et al., 2005).
The metabolic ratio for XO activity remained unaltered across the menstrual cycle. Only a few studies are available to date that report a lack of effect of the menstrual cycle on XO activity (Lane et al., 1992; Kashuba et al., 1998). Despite the paucity in the literature regarding the influence of menstrual cycle phases on XO activity, indirect data from studies on pregnant women (Tsutsumi et al., 2001), women taking OCs (Shelepova et al., 2005), or gender-related studies (Vistisen et al., 1992; Chung et al., 2000; Nowell et al., 2002; Aklillu et al., 2003; Begas et al., 2007) indicate lack of any effect of estrogens on the activity of XO.

The frequency distribution of metabolic ratios for slow $(27 / 42,64.3 \%)$ and fast $(15 / 42,35.7 \%)$ acetylators in the present study was in agreement with that previously reported for the Greek population (Asprodini et al., 1998; Begas et al., 2007). The metabolic ratio for NAT2 activity in slow acetylators was significantly higher at LFP compared with EFP and LP, whereas fast acetylators exhibited similar NAT2 activities among the three sampling phases. The lack of difference in slow acetylators between EFP and LP agrees 
A

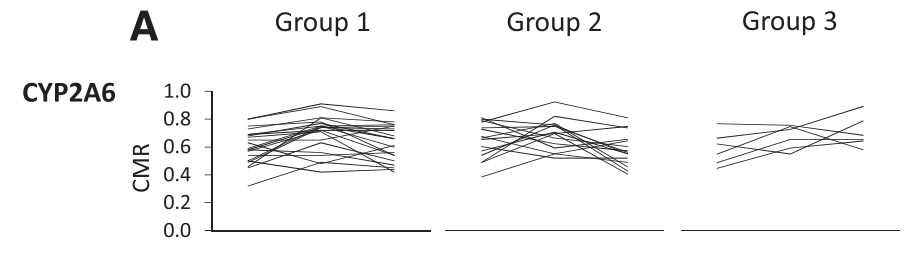

xo

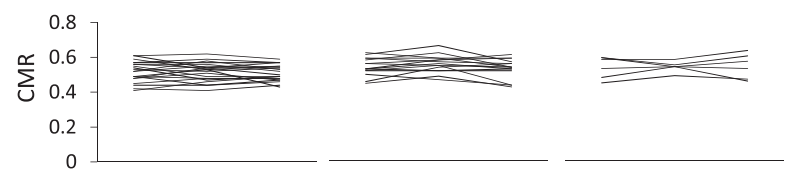

NAT2

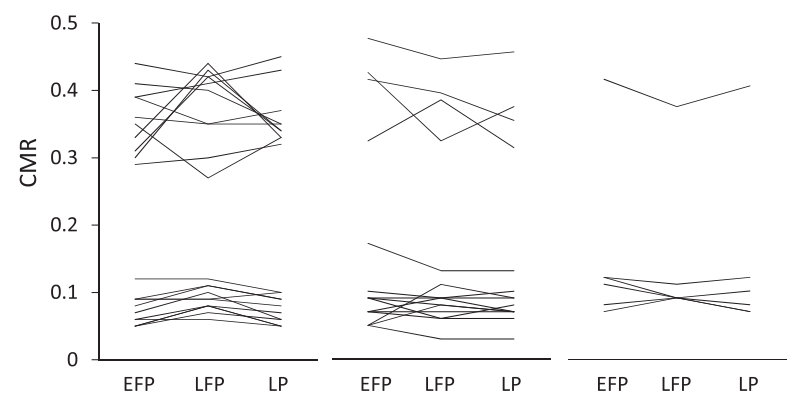

B
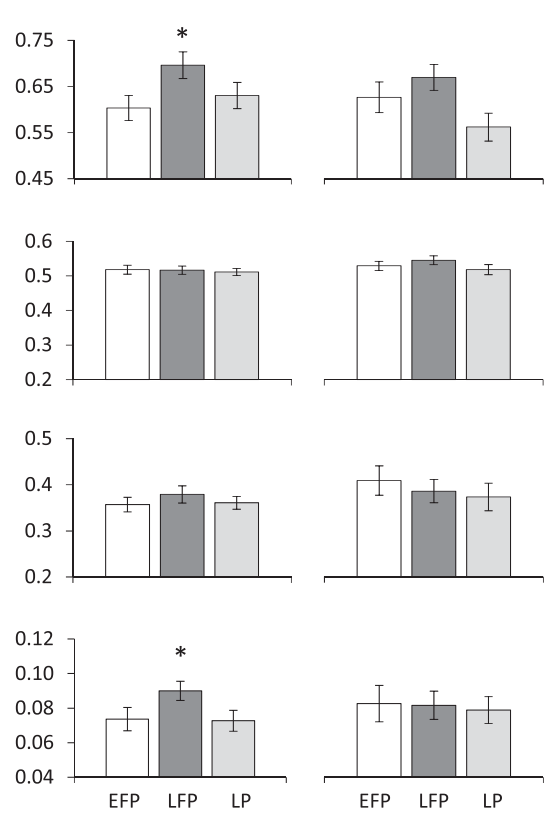

Group 2

Group 3
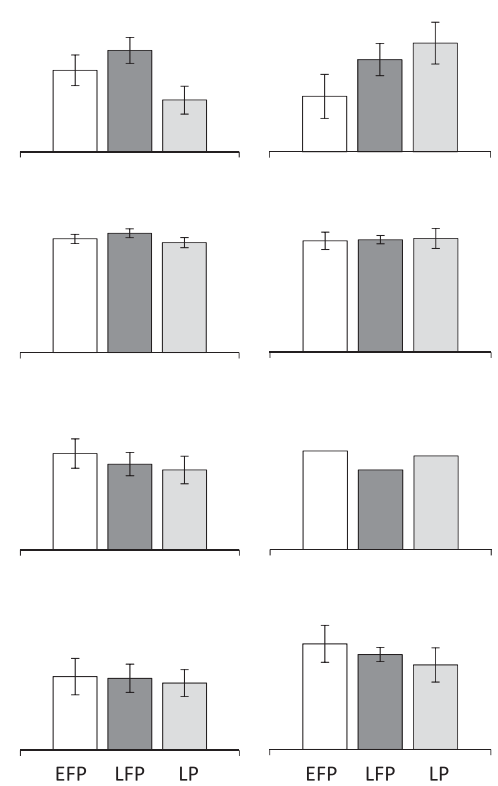

Fig. 5. CYP2A6, XO, and NAT2 in vivo indices during menstrual cycle. Individual data (A) and mean estimates ( \pm S.E.) (B) of CMRs measured in the three groups of volunteers (group $1, n=21$; group $2, n=15$; group $3, n=6$ ) at EFP, LFP, and LP. ${ }^{*} \mathrm{CYP} 2 \mathrm{~A} 6$ CMR at LFP is significantly higher compared with EFP $(P=0.002)$ and LP $(P=0.009)$ (one-way repeated-measures ANOVA). Similarly, NAT2 CMR (slow acetylators) at LFP is significantly higher compared with $\operatorname{EFP}(P=0.002)$ and $\operatorname{LP}(P=0.002)$ (one-way repeated-measures ANOVA).

with an earlier study reporting a lack of difference between the follicular and luteal phases in NAT2 activity (Kashuba et al., 1998). Studies on pregnant women (Vistisen et al., 1992; Tsutsumi et al., 2001) or women on estrogen therapy (Shelepova et al., 2005; O'Connell et al., 2006) reported no alteration of NAT2 activity in the presence of estrogens; these studies, however, based their conclusion on a limited number of subjects and, as expected, with low power for distinction between fast and slow acetylators.

Our study bears limitations, including the assessment of CYP1A2 activity over a single menstrual cycle, the use of an indirect method for detecting ovulation, and the lack of verification of caffeine abstinence by baseline sample analysis. Furthermore, despite the consent of participants to comply with the study protocol, the outpatient setting of our study precluded any rigid control over lifestyle factors influencing the activity of the enzymes studied, thus contributing to both intra- and interindividual variation. The considerable variability in altered CYP1A2 metabolic ratios in LFP ranging from $+26.3 \%$ to $-52.9 \%$, in combination with the marked overlap of metabolic ratio values between nonsmokers and smokers, renders the prediction of CYP1A2 activity alteration among individuals difficult.

Variations in xenobiotic-metabolizing enzyme activity across the menstrual cycle may pose challenges in women in terms of drug efficacy and toxicity, adverse reactions, and potential drug-drug interactions, a major concern in medicine for both clinicians and patients. In addition to drug metabolism, women may be susceptible to variations in xenobiotic transformation of several carcinogenic and precarcinogenic compounds, such as nitrosamines, aflatoxins, and polycyclic aromatic hydrocarbons. The results of the present study suggest that sex-related physiologic factors may be an important variable in xenobiotic metabolism.
In conclusion, the present study provides evidence for significant alterations in drug-metabolizing in vivo enzyme activities across the menstrual cycle. Although the clinical impact of the present data remains to be determined, our study provides a better understanding of pharmacokinetic alterations during the menstrual cycle and forms a basis for future clinical investigations and optimization of drug therapy in women.

\section{Acknowledgments}

We thank all volunteers who participated in the study.

\section{Authorship Contributions}

Participated in research design: Asprodini, Messinis.

Conducted experiments: Tsiokou, Begas, Kouvaras, Samara.

Performed data analysis: Asprodini, Tsiokou, Begas, Kilindris,

Kouvaras.

Wrote or contributed to the writing of the manuscript: Asprodini, Tsiokou, Kilindris, Messinis.

\section{References}

Abbassi-Ghanavati M, Greer LG, and Cunningham FG (2009) Pregnancy and laboratory studies: a reference table for clinicians. Obstet Gynecol 114:1326-1331.

Aklillu E, Carrillo JA, Makonnen E, Bertilsson L, and Ingelman-Sundberg M (2003) Xanthine oxidase activity is influenced by environmental factors in Ethiopians. Eur J Clin Pharmacol 59:533-536.

Asprodini EK, Zifa E, Papageorgiou I, and Benakis A (1998) Determination of N-acetylation phenotyping in a Greek population using caffeine as a metabolic probe. Eur J Drug Metab Pharmacokinet 23:501-506.

Begas E, Kouvaras E, Tsakalof A, Papakosta S, and Asprodini EK (2007) In vivo evaluation of CYP1A2, CYP2A6, NAT-2 and xanthine oxidase activities in a Greek population sample by the RP-HPLC monitoring of caffeine metabolic ratios. Biomed Chromatogr 21:190-200.

Beischlag TV and Perdew GH (2005) ER alpha-AHR-ARNT protein-protein interactions mediate estradiol-dependent transrepression of dioxin-inducible gene transcription. J Biol Chem 280:21607-21611.

Benowitz NL, Lessov-Schlaggar CN, Swan GE, and Jacob P, III (2006) Female sex and oral contraceptive use accelerate nicotine metabolism. Clin Pharmacol Ther 79:480-488.

Benowitz NL, Peng M, and Jacob P, III (2003) Effects of cigarette smoking and carbon monoxide on chlorzoxazone and caffeine metabolism. Clin Pharmacol Ther 74: $468-474$. 
Bruguerolle B, Toumi M, Faraj F, Vervloet D, and Razzouk H (1990) Influence of the menstrual cycle on theophylline pharmacokinetics in asthmatics. Eur J Clin Pharmacol 39:59-61.

Chang S-Y, Chen C, Yang Z, and Rodrigues AD (2009) Further assessment of 17alpha-ethinyl estradiol as an inhibitor of different human cytochrome P450 forms in vitro. Drug Metab Dispos 37:1667-1675.

Choi S-Y, Koh KH, and Jeong H (2013) Isoform-specific regulation of cytochromes P450 expression by estradiol and progesterone. Drug Metab Dispos 41:263-269.

Chung WG, Kang JH, Park CS, Cho MH, and Cha YN (2000) Effect of age and smoking on in vivo CYP1A2, flavin-containing monooxygenase, and xanthine oxidase activities in Koreans: determination by caffeine metabolism. Clin Pharmacol Ther 67:258-266.

Dempsey D, Jacob P, III, and Benowitz NL (2002) Accelerated metabolism of nicotine and cotinine in pregnant smokers. J Pharmacol Exp Ther 301:594-598.

Dobrinas M, Cornuz J, Oneda B, Kohler Serra M, Puhl M, and Eap CB (2011) Impact of smoking, smoking cessation, and genetic polymorphisms on CYP1A2 activity and inducibility. Clin Pharmacol Ther 90:117-125.

Evans DA (1989) N-acetyltransferase. Pharmacol Ther 42:157-234.

Faber MS, Jetter A, and Fuhr U (2005) Assessment of CYP1A2 activity in clinical practice: why, how, and when? Basic Clin Pharmacol Toxicol 97:125-134.

Fuhr U, Klittich K, and Staib AH (1993) Inhibitory effect of grapefruit juice and its bitter principal, naringenin, on CYP1A2 dependent metabolism of caffeine in man. Br J Clin Pharmacol 35:431-436.

Granfors MT, Backman JT, Laitila J, and Neuvonen PJ (2005) Oral contraceptives containing ethinyl estradiol and gestodene markedly increase plasma concentrations and effects of tizanidine by inhibiting cytochrome P450 1A2. Clin Pharmacol Ther 78:400-411.

Graziottin A and Serafini A (2016) Perimenstrual asthma: from pathophysiology to treatment strategies. Multidiscip Respir Med 11:30.

Gu L, Gonzalez FJ, Kalow W, and Tang BK (1992) Biotransformation of caffeine, paraxanthine, theobromine and theophylline by cDNA-expressed human CYP1A2 and CYP2E1. Pharmacogenetics 2:73-77.

Gunes A, Ozbey G, Vural EH, Uluoglu C, Scordo MG, Zengil H, and Dahl M-L (2009) Influence of genetic polymorphisms, smoking, gender and age on CYP1A2 activity in a Turkish population. Pharmacogenomics 10:769-778.

Hakooz NMK (2009) Caffeine metabolic ratios for the in vivo evaluation of CYP1A2, $\mathrm{N}$-acetyltransferase 2, xanthine oxidase and CYP2A6 enzymatic activities. Curr Drug Metab 10:329-338.

Hambridge HL, Mumford SL, Mattison DR, Ye A, Pollack AZ, Bloom MS, Mendola P, Lynch KL, Wactawski-Wende J, and Schisterman EF (2013) The influence of sporadic anovulation on hormone levels in ovulatory cycles. Hum Reprod 28: $1687-1694$

Herzog AG (2008) Catamenial epilepsy: definition, prevalence pathophysiology and treatment. Seizure 17:151-159.

Hukkanen J, Gourlay SG, Kenkare S, and Benowitz NL (2005) Influence of menstrual cycle on cytochrome P450 2A6 activity and cardiovascular effects of nicotine. Clin Pharmacol Ther 77:159-169.

Kall MA, Vang O, and Clausen J (1996) Effects of dietary broccoli on human in vivo drug metabolizing enzymes: evaluation of caffeine, oestrone and chlorzoxazone metabolism. Carcinogenesis 17:793-799.

Kamimori GH, Joubert A, Otterstetter R, Santaromana M, and Eddington ND (1999) The effect of the menstrual cycle on the pharmacokinetics of caffeine in normal, healthy eumenorrheic females. Eur J Clin Pharmacol 55:445-449.

Karjalainen MJ, Neuvonen PJ, and Backman JT (2008) In vitro inhibition of CYP1A2 by model inhibitors, anti-inflammatory analgesics and female sex steroids: predictability of in vivo interactions. Basic Clin Pharmacol Toxicol 103:157-165.

Kashuba ADM, Bertino JS, Jr, Kearns GL, Leeder JS, James AW, Gotschall R, and Nafziger AN (1998) Quantitation of three-month intraindividual variability and influence of sex and menstrual cycle phase on CYP1A2, N-acetyltransferase-2, and xanthine oxidase activity determined with caffeine phenotyping. Clin Pharmacol Ther 63:540-551.

Konstandi M, Cheng J, and Gonzalez FJ (2013) Sex steroid hormones regulate constitutive expression of Cyp2e1 in female mouse liver. Am J Physiol Endocrinol Metab 304:E1118-E1128

Lampe JW, King IB, Li S, Grate MT, Barale KV, Chen C, Feng Z, and Potter JD (2000) Brassica vegetables increase and apiaceous vegetables decrease cytochrome P450 1A2 activity in humans: changes in caffeine metabolite ratios in response to controlled vegetable diets. Carcinogenesis 21:1157-1162.

Lane JD, Steege JF, Rupp SL, and Kuhn CM (1992) Menstrual cycle effects on caffeine elimination in the human female. Eur J Clin Pharmacol 43:543-546.

Lelo A, Miners J0, Robson RA, and Birkett DJ (1986) Quantitative assessment of caffeine partial clearances in man. Br J Clin Pharmac 22:183-186.

Lew KH, Ludwig EA, Milad MA, Donovan K, Middleton E, Jr, Ferry JJ, and Jusko WJ (1993) Gender-based effects on methylprednisolone pharmacokinetics and pharmacodynamics. Clin Pharmacol Ther 54:402-414.
Nagata K, Ishitobi K, Yamamoto Y, Ikeda T, Hori S, Matsumoto Y, and Sasaki T (1997) Increased theophylline metabolism in the menstrual phase of healthy women. J Allergy Clin Immunol 100:39-43.

Nazir S, Iqbal Z, Ahmad L, Shah Y, and Nasir F (2015) Pharmacokinetics of omeprazole and its metabolites in three phases of menstrual cycle. Eur J Drug Metab Pharmacokinet 40:13-22.

Nowell S, Sweeney C, Hammons G, Kadlubar FF, and Lang NP (2002) CYP2A6 activity determined by caffeine phenotyping: association with colorectal cancer risk. Cancer Epidemiol Biomarkers Prev 11:377-383.

O'Connell MB, Frye RF, Matzke GR, St Peter JV, Willhite LA, Welch MR, Kowal P, and LaValleur J (2006) Effect of conjugated equine estrogens on oxidative metabolism in middle-aged and elderly postmenopausal women. J Clin Pharmacol 46:1299-1307.

Perera V, Gross AS, and McLachlan AJ (2012) Influence of environmental and genetic factors on CYP1A2 activity in individuals of South Asian and European ancestry. Clin Pharmacol Ther 92:511-519.

Peterson S, Schwarz Y, Li SS, Li L, King IB, Chen C, Eaton DL, Potter JD, and Lampe JW (2009) CYP1A2, GSTM1, and GSTT1 polymorphisms and diet effects on CYP1A2 activity in a crossover feeding trial. Cancer Epidemiol Biomarkers Prev 18:3118-3125.

Pollock BG, Wylie M, Stack JA, Sorisio DA, Thompson DS, Kirshner MA, Folan MM, and Condifer KA (1999) Inhibition of caffeine metabolism by estrogen replacement therapy in postmenopausal women. J Clin Pharmacol 39:936-940.

Prior JC, Naess M, Langhammer A, and Forsmo S (2015) Ovulation prevalence in women with spontaneous normal-length menstrual cycles - a population-based cohort from HUNT3, Norway. PLoS One 10:e0134473.

Reape KZ, DiLiberti CE, Hendy CH, and Volpe EJ (2008) Effects on serum hormone levels of low-dose estrogen in place of placebo during the hormone-free interval of an oral contraceptive. Contraception 77:34-39.

Reed BG and Carr BR (2000) The normal menstrual cycle and the control of ovulation, in Endotext (De Groot LJ, Chrousos G, Dungan K, Feingold KR, Grossman A, Hershman JM, Koch C, Korbonits M, McLachlan R, New M, et al. eds), MDText. com, Inc., South Dartmouth, MA

Sachse C, Brockmöller J, Bauer S, and Roots I (1999) Functional significance of a C-- $>$ A polymorphism in intron 1 of the cytochrome P450 CYP1A2 gene tested with caffeine. Br J Clin Pharmacol 47:445-449.

Schliep KC, Schisterman EF, Wactawski-Wende J, Perkins NJ, Radin RG, Zarek SM, Mitchell EM, Sjaarda LA, and Mumford SL (2016) Serum caffeine and paraxanthine concentrations and menstrual cycle function: correlations with beverage intakes and associations with race, reproductive hormones, and anovulation in the BioCycle study. Am J Clin Nutr 104:155-163.

Shavit G, Lerman P, Korczyn AD, Kivity S, Bechar M, and Gitter S (1984) Phenytoin pharmacokinetics in catamenial epilepsy. Neurology 34:959-961.

Shelepova T, Nafziger AN, Victory J, Kashuba ADM, Rowland E, Zhang Y, Sellers E, Kearns G, Leeder JS, Gaedigk A, et al. (2005) Effect of a triphasic oral contraceptive on drug-metabolizing enzyme activity as measured by the validated Cooperstown 5+1 cocktail. J Clin Pharmacol 45:1413-1421.

Tracy TS, Venkataramanan R, Glover DD, and Caritis SN; National Institute for Child Health and Human Development Network of Maternal-Fetal-Medicine Units (2005) Temporal changes in drug metabolism (CYP1A2, CYP2D6 and CYP3A activity) during pregnancy. Am J Obstet Gynecol 192:633-639.

Tsutsumi K, Kotegawa T, Matsuki S, Tanaka Y, Ishii Y, Kodama Y, Kuranari M, Miyakawa I, and Nakano S (2001) The effect of pregnancy on cytochrome P4501A2, xanthine oxidase, and N-acetyltransferase activities in humans. Clin Pharmaco Ther 70:121-125.

Vistisen K, Poulsen HE, and Loft S (1992) Foreign compound metabolism capacity in man measured from metabolites of dietary caffeine. Carcinogenesis 13:1561-1568.

Wilson K, Oram M, Horth CE, and Burnett D (1982) The influence of the menstrual cycle on the metabolism and clearance of methaqualone. Br J Clin Pharmacol 14:333-339.

Wójcicki J, Gawrońska-Szklarz B, Kazimierczyk J, Baskiewicz Z, and Raczyński A (1979) Comparative pharmacokinetics of paracetamol in men and women considering follicular and luteal phases. Arzneimittelforschung 29:350-352.

Wong P, Villeneuve G, Tessier V, Banerjee K, Nedev H, Jean-Claude BJ, and LeylandJones B (2002) Stability of 5-acetamido-6-formylamino-3-methyluracil in buffers and urine. J Pharm Biomed Anal 28:693-700.

Yamazaki H, Shaw PM, Guengerich FP, and Shimada T (1998) Roles of cytochromes $\mathrm{P} 4501 \mathrm{~A} 2$ and $3 \mathrm{~A} 4$ in the oxidation of estradiol and estrone in human liver microsomes. Chem Res Toxicol 11:659-665.

Zaigler M, Rietbrock S, Szymanski J, Dericks-Tan JS, Staib AH, and Fuhr U (2000) Variation of CYP1A2-dependent caffeine metabolism during menstrual cycle in healthy women. Int J Clin Pharmacol Ther 38:235-244.

Address correspondence to: E. Asprodini, Laboratory of Pharmacology, Faculty of Medicine, School of Health Sciences, University of Thessaly, 3 Panepistimiou, Biopolis, 41500 Larissa, Greece. E-mail: easpro@med.uth.gr 